\title{
Resolution Limitations from Detector Pulse Width and Jitter in a Linear Orthogonal-Acceleration Time-of-Flight Mass Spectrometer
}

\author{
J. N. Coles and M. Guilhaus \\ School of Chemistry, The University of New South Wales, Sydney, 2052, Australia
}

\begin{abstract}
Recent and ongoing advances in timing electronics together with the development of ionization techniques suited to time-of-flight mass spectrometry (TOF-MS) have contributed to renewed interest in this method of mass analysis. Whereas low resolving powers $(m / \Delta m$ $<500$ ) were once an almost unavoidable drawback in TOF-MS, recent developments in instrument geometries have produced much higher resolving powers for many ion sources. The temporal width of detector pulses and jitter in timing electronics, however, lead to contributions to peak widths that are essentially independent of the mass-analyzer ion optics. The effective detector pulse width $\left(\Delta t_{\mathrm{d}} \approx 1-10 \mathrm{~ns}\right.$ typically) can be a limiting factor in the development of high resolution time-of-flight (TOF) instruments with modest drift lengths $(\sim 1 \mathrm{~m})$. It also reduces the mass resolution more seriously for light ions. This article presents a method for distinguishing the instrumental "ion arrival-time" resolution $\left(R_{\mathrm{o}}\right)$ of a linear TOF mass analyzer from that which is locally measured at a particular mass, limited by the broadening of the detector pulse width and electronics. The method also provides an estimate of $\Delta t_{\mathrm{d}}$, that is useful in determining the temporal performance of the detection system. The model developed here is tested with data from a recently constructed orthogonal-acceleration TOF mass spectrometer equipped with a commercially available transient recorder (a LeCroy 400-Msample/s digital oscilloscope) from which we obtained $R_{0}=$ $4240 \pm 100$ [full width at half maximum (FWHM)] and $\Delta t_{\mathrm{d}}=3.0 \pm 0.1 \mathrm{~ns}$ (FWHM). (J Am Soc Mass Spectrom 1994, 5, 772-778)
\end{abstract}

$\mathrm{R}$ ecent developments in time-of-flight mass spectrometry (TOF-MS) have, for a range of ion sources, made available mass resolving powers well in excess of those attainable two decades ago [1-8]. This, together with advances in focal-plane ion detectors and digital timing electronics, is contributing to renewed interest in TOF-MS as an alternative to conventional scanning mass analyzers [9]. The ability of modern electronics to record ions from every dutycycle in time-of-flight (TOF) instruments with repetition rates in the kilohertz regime allows the intrinsic sensitivity advantage of TOF-MS over scanned instruments to be exploited.

For continuous ion sources, orthogonal-acceleration TOF-MS provides a good solution to the long-standing problems of the dispersions in initial ion parameters that affect the time-of-flight, namely, temporal, spatial, and energetic $[1,2,5,7,8]$. For our orthogonal-acceleration instrument $[1,2]$ it is now demonstrable that the width in time of the detector pulse is a significant limitation to the attainable mass resolving power.

Address reprint requests to Dr. M. Guilhaus, School of Chemistry, University of New South Wales, Sydney, 2052, Australia.
Moreover, as will be shown in this article, the detector pulse and jitter in the detection system generally lead to a decrease in resolving power as the mass is reduced.

This article examines the effect of a detection system based on a microchannel plate detector in conjunction with a commercially available transient recorder (a LeCroy 400-Msample/s digital oscilloscope) on the resolving power of a linear orthogonal-acceleration instrument. It offers a method to quantify the contribution of the detector and timing electronics to peak width. The analysis also shows that instruments with this geometry have a mass-independent resolving power based on the arrival-time spread of the ions at the detector.

\section{Experimental}

The experimental arrangement is shown in Figure 1. This prototype was built in close accordance with the specifications given in the earlier theoretical study [1]. The instrument at its present stage of completion has been described in detail elsewhere [2] and need not be 
discussed at length here. The ion source used was a VG 70-70 electron ionization/chemical ionization (VG Analytical Ltd., Manchester, UK) source operating with an ion extraction voltage of approximately $-60 \mathrm{~V}$. The filament current was trap-regulated at $50 \mu \mathrm{A}$ by an emission control unit, and the ion beam was collimated and decelerated to $14 \mathrm{eV}$ with suitable electrostatic lenses as it entered the orthogonal-acceleration region of the mass spectrometer.

A "push-out pulse" of $88 \mathrm{~V}$ with rise time of $\sim 100$ $\mathrm{ns}$ and a duration of $5 \mu \mathrm{s}$ in a total duty cycle of $97 \mu \mathrm{s}$ was applied to an electrode parallel to and $1.25 \mathrm{~mm}$ behind the beam axis. The beam passed through the first grid (5.3 mm from the push-out electrode), which was nominally at ground potential, and the next grid (5.5 mm from the first grid), which was maintained at $-101.5 \mathrm{~V}$ constantly. The final grid $(12.0 \mathrm{~mm}$ from the second grid) was set to $-2858 \mathrm{~V}$ and the ions traveled a total of $1504.2 \mathrm{~mm}$ along the drift tube to the $\mathrm{mi}$ crochannel plate (MCP) detector: a 25-mm-diameter Galileo-type $3025 \mathrm{~N}$ metal anode (Galileo Electro-Optics Sturbridge, MA). The same potential was applied to

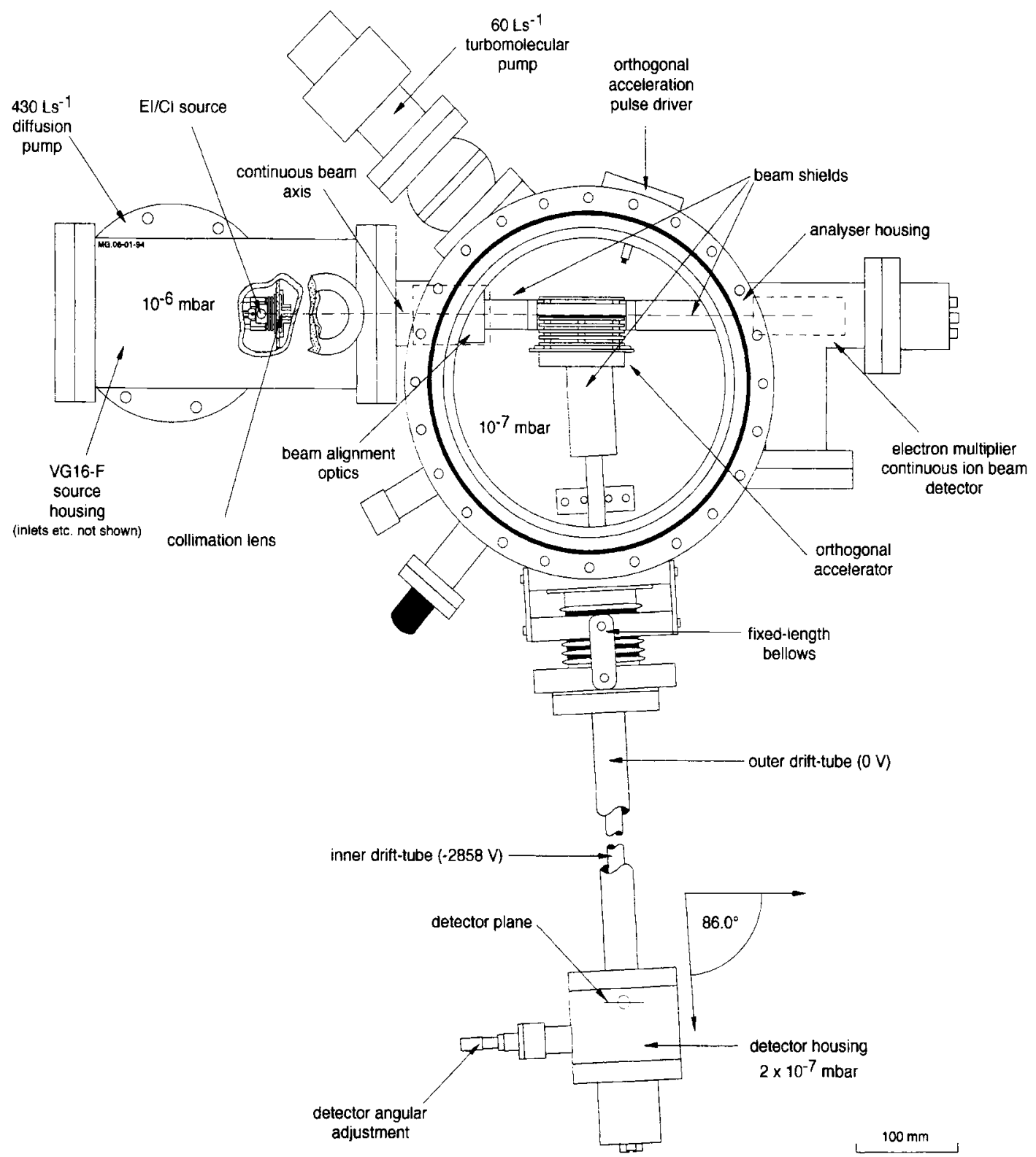

Figure 1. Arrangement of the orthogonal-acceleration time-of-flight mass spectrometer used in this work. 
the front of the MCP as was to the final grid and an insulated drift tube within the vacuum system.

We have constructed for the MCP a conical anode of approximately $50-\Omega$ impedance [10-12] to match the 50- $\Omega$ input of a LeCroy (Chestnut Ridge, NY) 9450 digital oscilloscope with a bandwidth limit of 350 $\mathrm{MHz}$. The oscilloscope was capable of sum-averaging up to $10^{6}$ traces with a time resolution of $2.5 \mathrm{~ns}$ in the normal mode or to $0.1 \mathrm{~ns}$ in the random-interleave sampling mode (RIS). The MCP detector pulses were applied directly to the LeCroy 9450 , which was also able to measure the time-of-flight of any peak in the spectrum to within $\pm 0.05 \mathrm{~ns}$, but a slight impedance mismatch, as yet uncorrected, is responsible for some $\pm 2 \%$ postpeak "ringing" on the baseline for about 50 ns. All peak width measurements were performed on data obtained in the RIS mode with the maximum time resolution of $0.1 \mathrm{~ns}$.

\section{Mass Resolving Power}

The peak width in magnetic spectrometers increases with mass in such a way that usually the measured resolution is the same at any mass number. In TOF mass spectrometers such as ours $[1,2]$ this is not the case, and a rigorous mathematical assessment is needed to interpret the variation of resolving power that is measured through the spectrum.

In a TOF mass analyzer a sample of ions of mass $m$ (kilograms) and charge $q=z e$ (Coulombs) is accelerated through a potential difference $V$ (volts) to attain a velocity $v$ (meters per second), such that their kinetic energy is

$$
\frac{1}{2} m v^{2}=q V
$$

so that the velocity of the ions is

$$
v=\sqrt{\frac{2 q V}{m}}
$$

For a drift region of length $D$ (meters) the time-offlight, $t$ (TOF) spent in the drift region is

$$
t=\frac{D}{v}
$$

and so from eq 1 we obtain

$$
t=D \sqrt{\frac{m}{2 q V}}
$$

We are more interested in the total time-of-flight and therefore have defined $D^{\prime}$ to be the effective drift length. This is the distance that the ions would have to travel at their final drift velocity $v$ (meters per second), free of initial spatial, velocity, and temporal displacement, to incur the same total flight-time $t$ (seconds) that similar ions would actually incur when accelerated from rest into the mass spectrometer. In our apparatus the exact drift-region length is $D=1.5042$ $\mathrm{m}$ and the effective drift length $D^{\prime}=1.6027 \mathrm{~m}$, which is independent of mass.

In our linear instrument, and undoubtedly in many others, the major contributor to ion arrival-time spread at the detector is the spread in final velocities (in the time-of-flight direction) of isobaric ions that originate from points equidistant from the plane of the detector. Typically this velocity spread can have the following sources:

1. Initial ion velocity (usually of thermal origin).

2. Deflection of ions as they experience field inhomogeneities in the vicinity of meshes.

3. Instability of voltages used to produce the electric fields for ion acceleration.

Whatever the relative contributions from these and other sources prior to the drift region, they amount to a mass-independent spread of kinetic energy. In eq 2 , this is analogous to a finite spread of $V$.

Differentiating eq 2 with respect to $V$ gives

$$
\frac{d t}{d V}=-\frac{D^{\prime}}{2} \sqrt{\frac{m}{2 q}} V^{-3 / 2}
$$

so that for a small but finite energy spread $q \Delta V$ there is a corresponding spread in arrival time $\Delta t_{\mathrm{a}}$ at the detector given by

$$
\Delta t_{\mathrm{a}}=-\frac{D^{\prime}}{2} \sqrt{\frac{m}{2 q}} V^{-3 / 2} \Delta V
$$

or

$$
\Delta t_{\mathrm{a}}=-\frac{D^{\prime} \Delta V}{2 V} \sqrt{\frac{m}{2 q V}}
$$

Combining eqs 2 and 3 we also note that a simple relationship holds:

$$
\frac{t}{2 \Delta t}=-\frac{V}{\Delta V}
$$

Now differentiating eq 2 with respect to mass we obtain

$$
\frac{d t}{d m}=\frac{D^{\prime}}{2} \frac{m^{-1 / 2}}{\sqrt{2 q V}}
$$

which, for small and finite increments of mass $\Delta m$ and time $\Delta t$, can be written

$$
\Delta t=\frac{D^{\prime}}{2} \sqrt{\frac{m}{2 q V}} \frac{\Delta m}{m}
$$


Noting that the definition of mass resolving power is $R=m / \Delta m$, where $\Delta m$ is the peak full width at half maximum (FWHM), then eq 5 gives

$$
R=\frac{D^{\prime}}{2 \Delta t} \sqrt{\frac{m}{2 q V}}
$$

From eqs 2,4 and 6 we can also state, therefore, that the resolving power is

$$
\frac{m}{\Delta m}=\frac{t}{2 \Delta t}
$$

that is

$$
R=\frac{t}{2 \Delta t}=\left|\frac{V}{\Delta V}\right|
$$

This suggests that the resolving power, based on the arrival-time spread of ions, is mass independent. Considering the peak width $\Delta t \approx 8.2 \mathrm{~ns}$ (FWHM) at $\mathrm{m} / \mathrm{z}$ 866 shown in Figure 2, which is an example taken from our instrument, the time-of-flight for the $m / z 866$ peak was measured accurately to be $62.9354 \mu \mathrm{s}$.

The local mass resolving power from eq 7 is

$$
R=\frac{t}{2 \Delta t}=\frac{62,935}{2 \times 8.2}=3860(\text { FWHM })
$$

However, under the same conditions, the resolving power at $\mathrm{m} / \mathrm{z} 40$ is about 2000 . We and other TOF investigators find that the locally measured mass resolution varies throughout the spectrum $[2,3,13]$.

\section{Ion Arrival-Time Spread and the Effect of Finite Detector Pulse Width}

A simple relationship between resolving power and the energy spread of the ions is given in eq 7. Our apparatus introduces minimal temporal spread be-

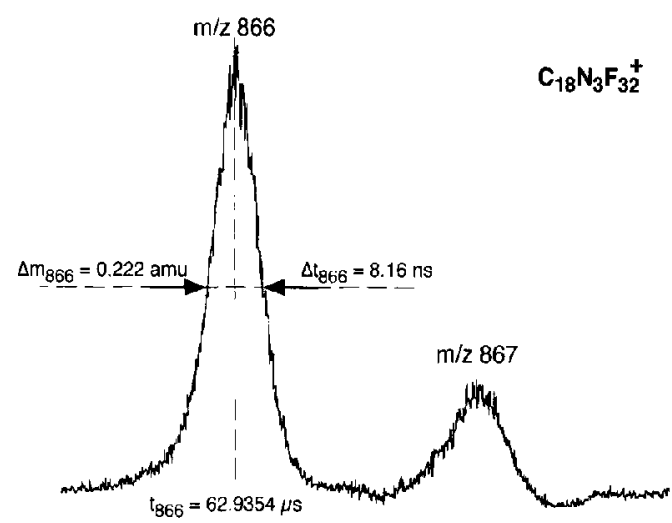

Figure 2. Peaks at $m / z 866$ and 867 in the $70-\mathrm{eV}$ positive-ion mass spectrum of tris-2,4,6-(pentadecafluoroheptyl)-1,3,5-triazine. This trace was recorded with a time base of $10 \mathrm{~ns} / \mathrm{div}$ (RIS) on a LeCroy 9450 digital ascilloscope, sum-averaged over 10,000 sweeps (equivalent to $1 \mathrm{~s}$ of data acquisition for the mass range $0-2200)$. cause the orthogonal gating is effectively instantaneous [2]. Ionization is continuous and once the ion beam has filled the orthogonal accelerator all ions begin their acceleration as the push-out electric field for a particular duty cycle is propagated. The rise time of the orthogonal-acceleration pulse is easily made much shorter than the time for a light ion to leave the first stage of the accelerator. Thus the acceleration is initially a simple function of time. This only slightly alters the space-focus condition compared to an idealized instantaneous and then static acceleration. In fact it is the irreproducibility of the attack (shape of the onset) of the pulse together with instability of its amplitude that can give rise to a small temporal spread in this gating method.

If we wish to describe the resolution on the basis of arrival time only then we can use eq 6 with $\Delta t$ replaced by $\Delta t_{\mathrm{a}}$ to define the "arrival-time" (only) optimum mass resolving power, $R_{0}$, which then becomes

$$
R_{\mathrm{o}}=\frac{D^{\prime}}{2 \Delta t_{\mathrm{a}}} \sqrt{\frac{m}{2 q V}}
$$

or

$$
\Delta t_{\mathrm{a}}=\frac{D^{\prime}}{2 R_{\mathrm{o}}} \sqrt{\frac{m}{2 q V}}
$$

From eq 9 it is apparent that the increment of arrivaltime spread is mass dependent, whereas $R_{\mathrm{o}}$ is actually a constant for the instrument as we shall show. The measured mass resolving power of a TOF spectrometer, however, is not dependent only on the arrival-time spread at the detector, but also on the finite pulse width that is produced by an incoming ion at the detector and the limitations of the electronics thereafter to process that signal, which results in broadening of the pulse and "jitter." Together these effects increase the observed peak width $\Delta t_{\mathrm{m}}$ at a particular mass, so that the measurement is always greater than the actual arrival-time spread $\Delta t_{\mathrm{a}}$. We therefore define a time spread $\Delta t_{\mathrm{d}}$ attributable to the detector and electronics. The combination of the instrumental time spreads, $\Delta t_{\mathrm{a}}$ and $\Delta t_{\mathrm{d}}$, is not trivial. The two temporal distributions combine by convolution. The one-dimensional convolution integral [14] can be written with the two time functions $f(t)$ and $h(t)$ that describe the ion arrival-time distribution and detector single ion pulse, respectively:

$$
g(T)=\int_{-\infty}^{+\infty} f(t) h(T-t) d t
$$

For example, two square distributions (equal width) convolute into a triangular distribution that has twice the square distribution width at the base. The pulses from an electron multiplier or channel plate detector are typically not square, but are characterized by a finite rise time and fall time. Typically these pulses, 
after passing through the detection electronics, are better approximated by a Gaussian distribution than a square distribution. Peak shapes observed over a wide mass range in TOF-MS are usually approximately Gaussian and it would be reasonable to assume that the arrival-time distribution of ions is not far from normal. The convolution of two Gaussian functions produces a new Gaussian function with the widths adding in quadrature. This would suggest that $\Delta t_{\mathrm{a}}$ and $\Delta t_{\mathrm{d}}$ add as follows to give a peak width $\Delta t_{\mathrm{m}}$ at a particular mass:

$$
\Delta t_{\mathrm{m}}^{2}=\Delta t_{\mathrm{a}}^{2}+\Delta t_{\mathrm{d}}^{2}
$$

For most focal-plane ion detectors, $\Delta t_{\mathrm{d}}$ is in the range $1-10 \mathrm{~ns}$. For a practical TOF mass analyzer with a resolving power of more than several hundred, the low end of this range is desirable for low masses (where $\Delta t_{\mathrm{d}}>\Delta t_{\mathrm{a}}$ ) if resolving power is to be maintained without recourse to very long drift regions. At high mass (where $\Delta t_{\mathrm{d}} \ll \Delta t_{\mathrm{a}}$ the resolving power approaches $R_{\mathrm{o}}$; it is only slightly diminished by the detector and electronics. Employing a longer drift region certainly will diminish the relative contribution of the detector $\Delta t_{\mathrm{d}}$ [9], but from practical or commercial standpoints this is not desirable because it increases the scale of the instrument to place greater demands on the vacuum system and the detector size.

Direct measurement of the detector pulse and jitter $\Delta t_{\mathrm{d}}$ is technically difficult even with a very fast oscilloscope. A bandwidth of better than $1 \mathrm{GHz}$ is desirable for 2-ns detector pulses. Even with such a device, the statistical distribution of pulse amplitudes leads to a distribution of triggering positions (in time) when signal averaging and/or random interleaved sampling is employed. In such cases $\Delta t_{\mathrm{d}}$ can be substantially overestimated.

$R_{\mathrm{o}}$ is a useful and meaningful instrument constant that provides a measure of the mass analyzer performance that is separate from the performance of the detector and electronics. When the data of many peaks are considered we find that $R_{\mathrm{o}}$ can be estimated along with $\Delta t_{\mathrm{d}}$. Substituting eq 9 into 10 we obtain

$$
\Delta t_{\mathrm{m}}=\sqrt{\frac{D^{\prime 2} m}{8 R_{\mathrm{o}}^{2} q V}+\Delta t_{\mathrm{d}}^{2}}
$$

From eq 11 it is apparent that when $\Delta t_{\mathrm{d}} \ll \Delta t_{\mathrm{a}}$ (i.e., at high mass or when the time-of-flight is long due to a large drift length), the relationship between $\Delta t_{\mathrm{m}}$ and $\sqrt{m}$ is nearly linear and the gradient is inversely proportional to the resolving power. As $m \rightarrow 0, \Delta t_{\mathrm{m}} \rightarrow$ $\Delta t_{d}$, so a graph of measured peak widths $\Delta t$ against $\sqrt{m}$, which covers the low and high mass ranges, should reveal both $R_{\mathrm{o}}$ and $\Delta t_{\mathrm{d}}$. Eight experimental peak widths (FWHM) from our prototype that cover the mass range from $m / z=4$ to 1166 have been plot-

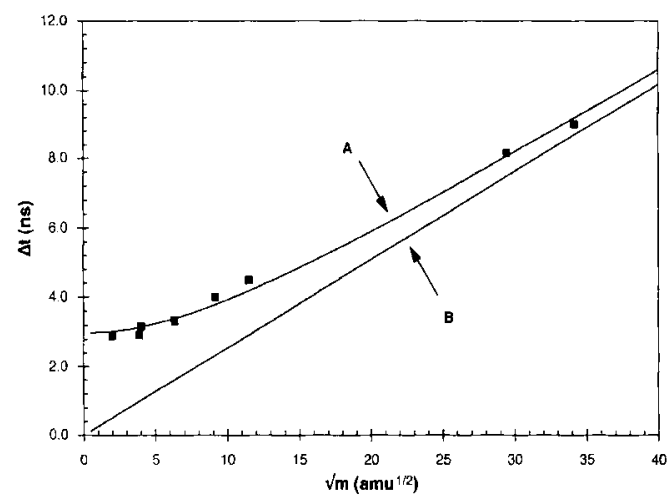

Figure 3. (a) The relationship between $\Delta t_{m}$ and $\sqrt{m}$ in the form of eq 11 with instrumental values for $V$ and $D^{\prime}$ and values chosen for $R_{\mathrm{o}}$ and $\Delta t_{\mathrm{d}}$ to satisfy the least-squares condition for the data points ( $\boldsymbol{D}$ ). (b) The relationship between $\Delta t_{\mathrm{a}}$ and $\sqrt{m}$ according to eq 9 and with the values for variables as determined for (a).

ted against $\sqrt{m}$ in Figure 3. A curve that conforms to the relationship of eq 11 was fitted to these points. Values for the two unknowns $\Delta t_{d}$ and $R_{\mathrm{o}}$ were chosen by minimizing the sum of the squares of the differences between (i) the experimental peak widths $\Delta t_{\exp _{i}}$ and (ii) $\Delta t_{m_{i^{\prime}}}$ those widths predicted by eq 11 :

$$
\sum_{i=1}^{8}\left\{\Delta t_{\exp _{i}}-\Delta t_{\mathrm{m}_{i}}\right\}^{2}=\text { minimum }
$$

This "least-squares" condition was met with

$$
R_{\mathrm{o}}=4243 ; \quad \Delta t_{\mathrm{d}}=2.99 \mathrm{~ns}
$$

The experimental points are in good agreement with the curve. The value for $\Delta t_{\mathrm{d}}$ is larger than the $\sim 2.0-\mathrm{ns}$ detector pulse width specified by the manufacturer for our pair of microchannel plates. If the width of the detector pulse was indeed $2.0 \mathrm{~ns}$, the time constant of the input amplifier of our $350-\mathrm{MHz}(-3-\mathrm{dB})$ oscilloscope would be expected to broaden this pulse by about $20 \%$ [15]. This leaves a discrepancy of 0.6 ns that reasonably could be accounted for by jitter in the ion-gating-timing electronics. The resolving power $R_{\mathrm{o}}$ is in reasonable agreement with earlier predictions [1].

We have tested the viability of these equations several times, because they are applicable throughout the mass spectrum, but are particularly important for light masses, where the term in $\Delta t_{\mathrm{d}}$ can be numerically more significant than $\Delta t_{\mathrm{a}}$ in determining the value of $R_{\mathrm{m}}$. This point is illustrated in the case of the spectrum of acetonitrile isobarically interfering with ${ }^{40} \mathrm{Ar}$ at $t=13,647.9 \mathrm{~ns}$ as shown in Figure 4. Here we observe a local mass resolving power of $R_{\mathrm{m}} \approx 2000$ (FWHM) and this agrees well with the value of 2010 


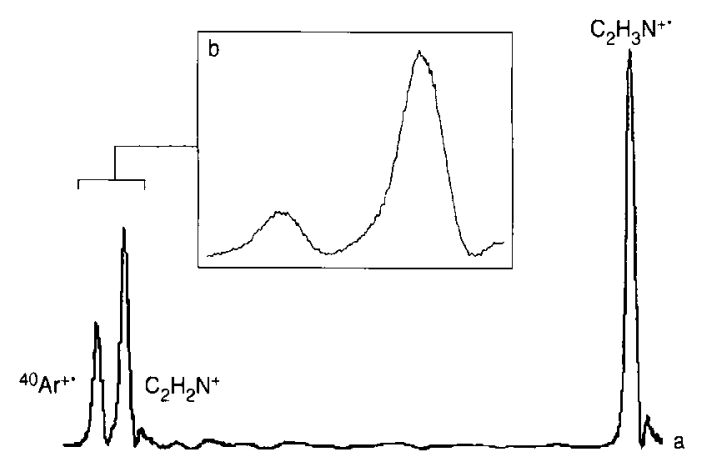

Figure 4. (a) The region from the $70-\mathrm{eV}$ positive-ion mass spectrum of acetonitrile $\left(\mathrm{C}_{2} \mathrm{H}_{3} \mathrm{~N}^{+}\right)$from a gas mixture also containing argon. (b) An expansion that shows the separation of the ${ }^{40} \mathrm{Ar}^{+}$peak from the fragment molecule $\mathrm{C}_{2} \mathrm{H}_{2} \mathrm{~N}^{+}$at $m / z \quad 40$ and the local mass resolving power at that mass, $R_{40} \approx 2000$ (FWHM).

from $t / 2 \Delta t_{\mathrm{m}}$ calculated by using $\Delta t_{\mathrm{m}}$ from eq 11 with the above-derived values for $R_{\mathrm{o}}$ and $\Delta t_{\mathrm{d}}$.

\section{Conclusions}

The temporal interaction of the detection system with the arrival of a packet of ions at the detector is by no means trivial. The model developed in this article separates the intrinsic resolving power of the mass analyzer, $R_{\mathrm{o}}$, from $\Delta t_{\mathrm{d}}$, a measure of the time resolution of the detection system. This has been useful to us because it provides a better basis for the choice of detector and a measure of the resolving power that is independent of the detection system. It also allows prediction of the resolving power at any mass of interest.

The title and discussion in this article are explicitly limited to linear orthogonal-acceleration TOF instruments. This is because the data available to us are from a device of that specific geometry. It is quite likely that the findings are relevant to other TOF-MS systems and in particular those employing the reflector geometry [16]. In the latter case the $\Delta V$ term of eq 3 is largely compensated, and $\Delta t_{\mathrm{a}}$ could remain small compared to $\Delta t_{d}$ up to a much higher mass than in a linear instrument.

\section{The Ideal Detection System}

Detector. Clearly a detector offering a smaller pulse width is desirable for high resolving power TOF-MS, particularly for the low mass range. Our experience is that current MCP technology offers the narrowest pulses for our high resolution needs at low mass $(m / z<500)$. For TOF instruments used primarily for detecting ions in the high mass range $(\mathrm{m} / z>1000)$ or where only a modest resolving power is required, larger values of $\Delta t_{\mathrm{d}}$ are tolerable. In such cases discrete dynode multipliers specifically designed for TOFMS [17] would probably be a better choice than MCPs because of their lower cost, higher durability, and lower dead time. This is particularly the case for instruments generating large fluxes of ions in each duty cycle (e.g., matrix-assisted laser desorption ionization TOF-MS).

It is likely that electron optics in ion detectors will present fundamental limits to the pulse width delivered and that in each case the conflicting requirements of narrow pulse width, high gain, and dynamic range will be balanced to meet the needs of the instrumentation.

Timing Electronics. Detection methods that measure current [e.g., integrating transient recorders (ITR)] pass on the full detector pulse width (typically $1-5 \mathrm{~ns}$ ) to the mass spectrum [18]. ITRs are now available that can signal average TOF mass spectra at repetition rates of more than $10 \mathrm{kHz}$ (transient recorder model 9825200, Precision Instruments, Knoxville, TN). A limitation of these devices is that they employ 8-bit flashconverter technology and multiplexing methods that combine to offer only a modest dynamic range $\left(10^{3}-10^{4}\right.$ limited either at high or low ion arrival rates). Even though signal averaging, in principle, can extend the dynamic range well above 8 bits, coherent noise that originates from the multiplex switching in these devices limits the dynamic range to about $10^{4}$. The use of nonlinear amplifiers (e.g., logarithmic) in conjunction with an ITR would provide improved dynamic range but the authors are not aware of any commercial devices with this feature.

Pulse-counting methods [e.g., time-to-digital converters (TDC)] detect the onset of detector pulses within a much smaller time window $(<1 \mathrm{~ns})$, but are limited because of ion-coincidence losses that give a low upper limit to the linear dynamic range [18]. It appears that until ITRs are available with higher resolution flash converters together with low-noise multiplexed accumulating memory, neither ITR nor TDC approaches will provide wide dynamic range in a high repetition-rate TOF instrument. A system that simultaneously has the attributes of a TDC and an ITR to cover a wide dynamic range would be useful. In this regard the authors note that LeBeyec and co-workers [19] have described a TDC system with a built-in fast analog-to-digital converter for measuring pulse height. Such a system provides a partial solution because it still is limited to the simultaneous arrival of only several ions.

Digitization Rate. This article has, until now, deliberately avoided the issue of the digitization rate of the detection electronics. This does not mean that it is unimportant. From the present discussion it is clear that higher digitization rates (e.g., > 500 Msample/s) are desirable, if not necessary, if resolving power is to be maintained at low mass. Fortunately, recent dramatic advances in the speed of timing electronics make 
Table 1. Comparison of cost and performance of LeCroy digital oscilloscopes in 1988 and 1994

\begin{tabular}{|c|c|c|}
\hline & LeCroy 9450 & LeCroy 9350 \\
\hline Year & 1988 & 1994 \\
\hline $\operatorname{Cost}^{a}$ & $\$ 35,000$ & $\$ 28,800$ \\
\hline Channels & 2 & 2 \\
\hline Record length ${ }^{b}$ & 50 Kpoints (12-bit) & 200 Kpoints (16-bit) \\
\hline Sampling speed ${ }^{3}$ & $400 \mathrm{Msample} / \mathrm{s}$ & $1 \mathrm{Gsample} / \mathrm{s}$ \\
\hline Bandwidth & $350 \mathrm{MHz}$ & $500 \mathrm{MHz}$ \\
\hline
\end{tabular}

\footnotetext{
australian currency.

- The data resolution is based on signal-averaged data. Single-shot data would have a 8-bit resolution in each case.

"The authors note that a four-channel $2-G s a m p l e / s$ option is available in the 9350 series but without a wider bandwidth. Because of the bandwidth fimitation, it is unlikely that the increased speed would be greatly advantageous in recording pulses of 1 -ns widths in TOF-MS.
}

available very fast digitizers. The cost and performance of these devices has fallen over the last decade as exemplified in Table 1, where the specifications of the commercial device used to acquire spectra in our laboratory are compared with a more recent device that has about the same cost. Although the sampling speed has increased by a factor of 2.5 , the cost has dropped by nearly $18 \%$. We therefore comment on the relative impact of digitization rate and detector pulse width and jitter as follows: The detector pulse width and jitter, particularly with ITR detection systems, currently present a more fundamental limitation of the resolving power attainable in TOF-MS than does the the achievement of high digitization rates. The latter is a technological problem with currently emerging solutions.

\section{Acknowledgments}

The financial assistance of the Australian Research Council and the Department of Industry, Technology and Commerce is gratefully acknowledged.

\section{References}

1. Dawson, J. H. J.; Guilhaus, M. Rapia Commun. Mass Spectrom. 1989, 3, 155-159.

2. Coles, J.; Guilhaus, M. Trends Anal. Chem. 1993, 12, 203-213.

3. Grix, R.; Kutsher, R.; Li, G.; Grüner U.; Wollnik, H. Rapid Conmun. Mass Spectrom., 1988, 2, 83-85.

4. Bergmann, T; Martin, T. P.; Shaber, H. Rev. Sci. Instrum. $1990,61,2585-2591$.

5. Boyle, J. G.; Whitehouse, C. M. Anal. Chem. 1992, 64, 2084-2089

6. Michael, S. M.; Chien, M.; Lubman, D. M. Rev. Sci. Instrum. 1992, 63, 4277-4284.

7. Mirgorodkaya, O. A.; Shevchenko, A. A.; Chernushevich, I. V.; Dodonov, A. F.; Miroshnikov, A. I. Anal. Chem. 1994, 66, 99-107.

8. Verentchikov, A.; Ens, W.; Standing, K. G. Abstracts and Proceedings of 41st ASMS Conference on Mass Spectrometry and Allied Topics; San Francisco, CA, May 30-June 4, 1993; paper MOA 10:50.

9. Cotter, R. J. Anal. Chem. 1992, 64, 1027A-1039A.

10. Pietri, G. IEEE Trans. Nucl. Sci. 1975, 22, 2084-2092.

11. Girard, J.; Balore, M. Nucl. Instrum. Methods 1977, 140, 279-282.

12. Kraus, R. H., Jr.; Viera, D. J.; Wollnik, H.; Wouters, J. M. Nucl. Instrum. Methods Phys. Res. 1988, A264, 327-332.

13. Cornish, T.; Cotter, R. J. Rapid Commun. Mass Spectrom. 1992, 6, 242-248.

14. Hecht, E.; Zajac, A. Optics; Addison-Wesley: Reading, MA, 1974; pp 406-411.

15. Characterising and Troubleshooting High Speed Circuits; Application Note ITI 015B; The LeCroy Corporation: Chestnut Ridge, NY, June 1993; p 4.

16. Mamyrin, B. A.; Shmikk, D. V. Sov. Phys. JETP 1979, 49, 762-764.

17. Active Film Multiplier Handbook for Mass Spectrometry; Technical publication TP004; ETP Scientific Inc.: 7 Midstate Drive, Auburn, MA, May 1993.

18. Ens, W.; Standing, K. G.; Verentchikov, A. Proceedings of the International Conference on Instrumentation for Time-offlight Mass Spectrometry, LeCroy Corporation, Chestnut Ridge, NY, 